\title{
Use of Selected Recommended Clinical Preventive Services - Behavioral Risk Factor Surveillance System, United States, 2018
}

\author{
Suhang Song, $\mathrm{PhD}^{1,2}$; Allison White ${ }^{1,2}$; James E. Kucik, $\mathrm{PhD}^{1}$
}

Clinical preventive services play an important role in preventing deaths, and Healthy People 2020 has set national goals for using clinical preventive services to improve population health (1). The Patient Protection and Affordable Care Act (ACA) requires many health plans to cover certain recommended clinical preventive services without cost-sharing when provided in-network (covered clinical preventive services).* To ascertain prevalence of the use of selected recommended clinical preventive services among persons aged $\geq 18$ years, CDC analyzed data from the 2018 Behavioral Risk Factor Surveillance System (BRFSS), a state-based annual nationwide survey conducted via landline and mobile phones in the United States, for 10 clinical preventive services covered in-network with no costsharing pursuant to the ACA. The weighted prevalence of colon, cervical, and breast cancer screening, pneumococcal and tetanus vaccination, and diabetes screening ranged from $66.0 \%$ to $79.2 \%$; the prevalence of the other four clinical preventive services were $<50 \%$ : $16.5 \%$ for human papillomavirus (HPV) vaccination, $26.6 \%$ for zoster (shingles) vaccination, $33.2 \%$ for influenza vaccination, and $45.8 \%$ for HIV testing. Prevalence of HIV testing had the widest variation (3.1-fold differences) across states among the 10 services included in this report. The prevalence of use of clinical preventive services varied by insurance status, income level, and rurality, findings that are consistent with previous studies (2-6). The use of nine of the 10 services examined was lower among the uninsured, those with lower income, and those living in rural communities. Among those factors examined, insurance status was the dominant factor strongly associated with use of clinical preventive services, followed by income-level and rurality. Understanding

\footnotetext{
*The Patient Protection and Affordable Care Act, Pub L. No. 111-148, 124 Stat. 131, Sect. 1001 (Mar. 23, 2010). The covered clinical preventive services were recommended by the U.S. Preventive Services Task Force, the Advisory Committee on Immunization Practices, and the Health Resources and Services Administration.
}

factors influencing use of recommended clinical preventive services can potentially help decision makers better identify policies to increase their use including strategies to increase insurance coverage.

\section{INSIDE}

467 Community-Associated Outbreak of COVID-19 in a Correctional Facility — Utah, September 2020January 2021

473 Willingness to Receive a COVID-19 Vaccination Among Incarcerated or Detained Persons in Correctional and Detention Facilities - Four States, September-December 2020

478 Rapid Spread of SARS-CoV-2 in a State Prison After Introduction by Newly Transferred Incarcerated Persons - Wisconsin, August 14-October 22, 2020

483 Counties with High COVID-19 Incidence and Relatively Large Racial and Ethnic Minority Populations — United States, April 1-December 22, 2020

490 Symptoms of Anxiety or Depressive Disorder and Use of Mental Health Care Among Adults During the COVID-19 Pandemic — United States,

August 2020-February 2021

495 Interim Estimates of Vaccine Effectiveness of BNT162b2 and mRNA-1273 COVID-19 Vaccines in Preventing SARS-CoV-2 Infection Among Health Care Personnel, First Responders, and Other Essential and Frontline Workers - Eight U.S. Locations, December 2020-March 2021

503 QuickStats

Continuing Education examination available at https://www.cdc.gov/mmwr/mmwr_continuingEducation.html

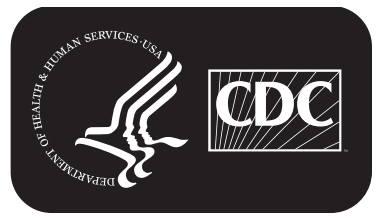

U.S. Department of Health and Human Services Centers for Disease Control and Prevention 
Six of the 10 recommended clinical preventive services that health plans are required to cover without cost-sharing were included in the 2018 BRFSS core questionnaire, which was used by all 50 states, the District of Columbia (DC), Guam, and Puerto Rico; these include colon, cervical, and breast cancer screening; HIV testing; and pneumococcal and influenza vaccination. The other four services were included in the optional modules, which are asked by some states; these include diabetes screening (asked by 28 states, DC, Guam, and Puerto Rico), HPV vaccination (asked by eight states), shingles vaccination (asked by four states), and tetanus vaccination (asked by four states). ${ }^{\dagger}$ Survey participants were classified as having used a clinical preventive service if they reported using a clinical preventive service as recommended at the time of interview. Because of changes over time to recommendations and to policies and practices that affect use of clinical preventive services, continued monitoring of their use could offer decision makers updated information for achieving public health goals.

In the 2018 BRFSS, the median survey response rate was $49.9 \%$ with a sample size of 437,436 adults aged $\geq 18$ years. Participants were considered uninsured if they didn't have any health care coverage at the time of the interview. Federal poverty level (FPL) was calculated by using the number of

\footnotetext{
$\dagger$ The BRFSS questionnaire has three parts: 1) the core component, 2) optional modules, and 3) state-added questions. Every state must ask the core component questions; however, the modules are optional. https://www.cdc.gov/brfss/ questionnaires/index.htm
}

adults, the number of children, and the midpoint income value of the categorical household income level ( 7 ). Persons with household income $\leq 138 \%$ of FPL as defined by the 2017 FPL threshold were categorized as lower income. BRFSS uses the 2013 CDC National Center for Health Statistics' UrbanRural Classification Scheme for Counties: urban counties are those coded as all four metropolitan categories plus micropolitan; rural counties are those coded as noncore. ${ }^{\S}$ Weighted utilization prevalence and $95 \%$ confidence intervals (CIs) are presented. Generalized linear modeling was used to estimate prevalence ratios (PRs) and 95\% CIs for the differences in use of clinical preventive services between persons in three categories: 1) insured versus uninsured, 2) higher versus lower income, and 3) rural versus urban residence. Subgroups were generated representing the interaction of these three variables, which resulted in eight insurance-income-residence combinations. Generalized linear modeling was also used to compare use of clinical preventive services use in each subgroup using STATA/MP (version 16; StataCorp), adjusted by age, sex, race/ethnicity, education, marital status, self-reported health status, and state.

Use varied across the 10 covered clinical preventive services (Table 1). The weighted prevalence of colon, cervical, and breast cancer screening, pneumococcal and tetanus vaccination, and diabetes screening ranged from $66.0 \%$ to $79.2 \%$; the

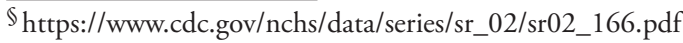

The MMWR series of publications is published by the Center for Surveillance, Epidemiology, and Laboratory Services, Centers for Disease Control and Prevention (CDC), U.S. Department of Health and Human Services, Atlanta, GA 30329-4027.

Suggested citation: [Author names; first three, then et al., if more than six.] [Report title]. MMWR Morb Mortal Wkly Rep 2021;70:[inclusive page numbers].

\section{Centers for Disease Control and Prevention Rochelle P. Walensky, MD, MPH, Director Anne Schuchat, MD, Principal Deputy Director Daniel B. Jernigan, MD, MPH, Acting Deputy Director for Public Health Science and Surveillance Rebecca Bunnell, PhD, MEd, Director, Office of Science Jennifer Layden, MD, PhD, Deputy Director, Office of Science Michael F. Iademarco, MD, MPH, Director, Center for Surveillance, Epidemiology, and Laboratory Services}

MMWR Editorial and Production Staff (Weekly)
Charlotte K. Kent, PhD, MPH, Editor in Chief Jacqueline Gindler, MD, Editor

Brian A. King, PhD, MPH, Guest Science Editor

Paul Z. Siegel, MD, MPH, Associate Editor

Mary Dott, MD, MPH, Online Editor

Terisa F. Rutledge, Managing Editor

Teresa M. Hood, MS, Acting Lead Technical Writer-Editor

Glenn Damon, Soumya Dunworth, PhD,

Catherine B. Lansdowne, MS, Srila Sen, MA,

Stacy Simon, MA, Jeffrey D. Sokolow, MA, Technical Writer-Editors

Matthew L. Boulton, MD, MPH Carolyn Brooks, ScD, MA Jay C. Butler, MD Virginia A. Caine, MD

Jonathan E. Fielding, MD, MPH, MBA David W. Fleming, MD
Martha F. Boyd, Lead Visual Information Specialist Alexander J. Gottardy, Maureen A. Leahy,

Julia C. Martinroe, Stephen R. Spriggs, Tong Yang, Visual Information Specialists

Quang M. Doan, MBA, Phyllis H. King,

Terraye M. Starr, Moua Yang,

Information Technology Specialists
Ian Branam, MA, Acting Lead

Health Communication Specialist Shelton Bartley, MPH,

Lowery Johnson, Amanda Ray,

Jacqueline N. Sanchez, MS,

Health Communication Specialists Will Yang, MA,

Visual Information Specialist

\footnotetext{
MMWR Editorial Board

Timothy F. Jones, MD, Chairman

William E. Halperin, MD, DrPH, MPH

Christopher M. Jones, PharmD, DrPH, MPH

Jewel Mullen, MD, MPH, MPA

Jeff Niederdeppe, $\mathrm{PhD}$

Celeste Philip, MD, MPH

Patricia Quinlisk, MD, MPH
} 
TABLE 1. Percentage of adults who received recommended clinical preventive services, by health insurance status, family income level, and rurality - Behavioral Risk Factor Surveillance System, United States, 2018

\begin{tabular}{|c|c|c|c|c|c|c|c|c|c|c|c|c|c|c|c|c|c|c|c|c|}
\hline \multirow{3}{*}{$\frac{\text { Characteristic }}{\text { Total }}$} & \multicolumn{20}{|c|}{ No. who received service, weighted $\%(95 \% \mathrm{Cl})$} \\
\hline & \multicolumn{2}{|c|}{$\begin{array}{l}\text { Colon cancer } \\
\text { screening, } \\
\text { age } 50-75 \text { yrs }\end{array}$} & \multicolumn{2}{|c|}{$\begin{array}{l}\text { Cervical cancer } \\
\text { screening } \\
\text { (women), } \\
\text { age 21-65 yrs }\end{array}$} & \multicolumn{2}{|c|}{$\begin{array}{l}\text { Breast cancer } \\
\text { screening } \\
\text { (within } 2 \text { yrs) } \\
\text { (women), } \\
\text { age } 50-74 \text { yrs }\end{array}$} & \multicolumn{2}{|c|}{$\begin{array}{l}\text { HIV testing } \\
\text { (ever), } \\
\text { age 18-65 yrs }\end{array}$} & \multicolumn{2}{|c|}{$\begin{array}{c}\text { Pneumococcal } \\
\text { vaccination } \\
\text { (ever), } \\
\text { age } \geq 65 \text { yrs }\end{array}$} & \multicolumn{2}{|c|}{$\begin{array}{c}\text { Influenza } \\
\text { vaccination } \\
\text { (within } 1 \mathrm{yr} \text { ), } \\
\text { age } \geq 18 \mathrm{yrs}\end{array}$} & \multicolumn{2}{|c|}{$\begin{array}{c}\text { Diabetes } \\
\text { screening } \\
\text { (within } 3 \text { yrs), } \\
\text { age } 40-70 \text { yrs }\end{array}$} & \multicolumn{2}{|c|}{$\begin{array}{c}\text { HPV vaccination } \\
\text { (ever), } \\
\text { age } 18-26 \text { yrs }\end{array}$} & \multicolumn{2}{|c|}{$\begin{array}{c}\text { Zoster } \\
\text { (shingles) } \\
\text { vaccination } \\
\text { (ever), } \\
\text { age } \geq 50 \text { yrs }\end{array}$} & \multicolumn{2}{|c|}{$\begin{array}{c}\text { Tetanus } \\
\text { vaccination } \\
\text { (within } \\
10 \text { yrs), } \\
\text { age } \geq 19 \text { yrs }\end{array}$} \\
\hline & 147,965 & $\begin{array}{c}68.4 \\
(67.9- \\
68.9)\end{array}$ & 106,362 & $\begin{array}{c}79.2 \\
(78.7- \\
79.6)\end{array}$ & 89,409 & $\begin{array}{c}78.7 \\
(78.2- \\
79.3)\end{array}$ & 113,284 & $\begin{array}{c}45.8 \\
(45.4- \\
46.2)\end{array}$ & 105,829 & $\begin{array}{c}71.0 \\
(70.4- \\
71.6)\end{array}$ & 164,092 & $\begin{array}{c}33.2 \\
(32.9- \\
33.5)\end{array}$ & 48,719 & $\begin{array}{c}68.8 \\
(68.0- \\
69.6)\end{array}$ & 527 & $\begin{array}{c}16.5 \\
(14.1- \\
18.9)\end{array}$ & 6,066 & $\begin{array}{c}26.6 \\
(25.3- \\
27.9)\end{array}$ & 17,390 & $\begin{array}{r}66.0 \\
(64.6- \\
67.4)\end{array}$ \\
\hline \multicolumn{21}{|c|}{ Insurance status } \\
\hline Insured & 143,667 & $\begin{array}{c}71.0 \\
(70.6- \\
71.5)\end{array}$ & 97,791 & $\begin{array}{c}81.0 \\
(80.6- \\
81.5)\end{array}$ & 86,525 & $\begin{array}{c}80.4 \\
(79.8- \\
81.0)\end{array}$ & 100,248 & $\begin{array}{c}46.1 \\
(45.7- \\
46.5)\end{array}$ & 104,463 & $\begin{array}{c}71.6 \\
(71.0- \\
72.2)\end{array}$ & 158,376 & $\begin{array}{c}35.9 \\
(35.6- \\
36.2)\end{array}$ & 45,840 & $\begin{array}{c}71.2 \\
(70.4- \\
72.0)\end{array}$ & 471 & $\begin{array}{c}19.1 \\
(16.3- \\
21.9)\end{array}$ & 5,928 & $\begin{array}{c}28.3 \\
(26.9- \\
29.7)\end{array}$ & 15,668 & $\begin{array}{c}69.4 \\
(68.1- \\
70.7)\end{array}$ \\
\hline Uninsured & 4,035 & $\begin{array}{c}34.1 \\
(32.1- \\
36.2)\end{array}$ & 8,343 & $\begin{array}{c}66.7 \\
(65.0- \\
68.5)\end{array}$ & 2,719 & $\begin{array}{c}54.2 \\
(51.2- \\
57.3)\end{array}$ & 12,639 & $\begin{array}{c}44.6 \\
(43.4- \\
45.8)\end{array}$ & 1,138 & $\begin{array}{c}43.9 \\
(38.8- \\
49.0)\end{array}$ & 5,303 & $\begin{array}{c}13.9 \\
(13.2- \\
14.7)\end{array}$ & 2,781 & $\begin{array}{c}49.8 \\
(46.5- \\
53.1)\end{array}$ & 54 & $\begin{array}{c}9.8 \\
(5.0- \\
14.6)\end{array}$ & 122 & $\begin{array}{c}9.5 \\
(5.9- \\
13.0)\end{array}$ & 1,666 & $\begin{array}{c}51.9 \\
(47.7- \\
56.1)\end{array}$ \\
\hline $\begin{array}{l}\text { Insured to } \\
\text { uninsured } \\
\text { prevalence } \\
\text { ratio* }\end{array}$ & $2.08^{\dagger}$ & $\begin{array}{c}(1.96- \\
2.21)\end{array}$ & $1.21^{\dagger}$ & $\begin{array}{c}(1.18- \\
1.25)\end{array}$ & $1.48^{\dagger}$ & $\begin{array}{c}(1.40- \\
1.57)\end{array}$ & $1.03^{\$}$ & $\begin{array}{c}(1.01- \\
1.06)\end{array}$ & $1.63^{\dagger}$ & $\begin{array}{c}(1.45- \\
1.83)\end{array}$ & $2.58^{\dagger}$ & $\begin{array}{c}(2.44- \\
2.72)\end{array}$ & $1.43^{\dagger}$ & $\begin{array}{c}(1.34- \\
1.53)\end{array}$ & $1.95^{\S}$ & $\begin{array}{c}(1.17- \\
3.25)\end{array}$ & $2.99^{\dagger}$ & $\begin{array}{c}(2.06- \\
4.35)\end{array}$ & $1.34^{\dagger}$ & $\begin{array}{c}(1.23- \\
1.45)\end{array}$ \\
\hline \multicolumn{21}{|l|}{ Income level } \\
\hline $\begin{array}{l}\text { Higher income } \\
\text { (income } \\
>138 \% \mathrm{FPL} \text { ) }\end{array}$ & 109,437 & $\begin{array}{c}71.8 \\
(71.2- \\
72.3)\end{array}$ & 71,638 & $\begin{array}{c}81.9 \\
(81.3- \\
82.4)\end{array}$ & 61,902 & $\begin{array}{c}80.7 \\
(80.0- \\
81.3)\end{array}$ & 74,501 & $\begin{array}{c}45.4 \\
(45.0- \\
45.9)\end{array}$ & 74,031 & $\begin{array}{c}73.5 \\
(72.9- \\
74.2)\end{array}$ & 116,176 & $\begin{array}{c}35.3 \\
(34.9- \\
35.7)\end{array}$ & 36,019 & $\begin{array}{c}70.4 \\
(69.5- \\
71.3)\end{array}$ & 309 & $\begin{array}{c}18.8 \\
(15.3- \\
22.2)\end{array}$ & 4,385 & $\begin{array}{c}29.2 \\
(27.6- \\
30.8)\end{array}$ & 11,825 & $\begin{array}{c}69.0 \\
(67.4- \\
70.5)\end{array}$ \\
\hline $\begin{array}{l}\text { Lower income } \\
\text { (income } \\
\leq 138 \% \mathrm{FPL} \text { ) }\end{array}$ & 16,938 & $\begin{array}{c}55.9 \\
(54.6- \\
57.2)\end{array}$ & 21,394 & $\begin{array}{c}75.5 \\
(74.4- \\
76.5)\end{array}$ & 11,966 & $\begin{array}{c}71.5 \\
(70.0- \\
72.9)\end{array}$ & 25,887 & $\begin{array}{c}51.8 \\
(51.0- \\
52.7)\end{array}$ & 10,647 & $\begin{array}{c}62.0 \\
(60.2- \\
63.8)\end{array}$ & 21,172 & $\begin{array}{c}26.6 \\
(25.9- \\
27.3)\end{array}$ & 7,263 & $\begin{array}{c}64.1 \\
(61.8- \\
66.4)\end{array}$ & 129 & $\begin{array}{c}15.6 \\
(10.6- \\
20.7)\end{array}$ & 499 & $\begin{array}{c}15.5 \\
(12.7- \\
18.2)\end{array}$ & 2,852 & $\begin{array}{c}60.5 \\
(57.0- \\
64.1)\end{array}$ \\
\hline $\begin{array}{l}\text { Higher to lower } \\
\text { income } \\
\text { prevalence } \\
\text { ratio* }\end{array}$ & $1.28^{\dagger}$ & $\begin{array}{c}(1.25- \\
1.32)\end{array}$ & $1.08^{\dagger}$ & $\begin{array}{c}(1.07- \\
1.10)\end{array}$ & $1.13^{\dagger}$ & $\begin{array}{c}(1.10- \\
1.15)\end{array}$ & $0.88^{\dagger}$ & $\begin{array}{c}(0.86- \\
0.89)\end{array}$ & $1.19^{\dagger}$ & $\begin{array}{c}(1.15- \\
1.22)\end{array}$ & $1.33^{\dagger}$ & $\begin{array}{c}(1.29- \\
1.36)\end{array}$ & $1.10^{\dagger}$ & $\begin{array}{c}(1.06- \\
1.14)\end{array}$ & 1.20 & $\begin{array}{c}(0.83- \\
1.74)\end{array}$ & $1.88^{\dagger}$ & $\begin{array}{c}(1.56- \\
2.27)\end{array}$ & $1.14^{\dagger}$ & $\begin{array}{c}(1.07- \\
1.21)\end{array}$ \\
\hline \multicolumn{21}{|l|}{ Rurality } \\
\hline Urban & 123,288 & $\begin{array}{c}68.9 \\
(68.4- \\
69.5)\end{array}$ & 89,873 & $\begin{array}{c}79.5 \\
(79.0- \\
79.9)\end{array}$ & 73,706 & $\begin{array}{c}79.0 \\
(78.4- \\
79.7)\end{array}$ & 97,576 & $\begin{array}{c}46.3 \\
(45.9- \\
46.7)\end{array}$ & 87,922 & $\begin{array}{c}71.7 \\
(71.1- \\
72.4)\end{array}$ & 138,216 & $\begin{array}{c}33.4 \\
(33.1- \\
33.7)\end{array}$ & 38,737 & $\begin{array}{c}68.5 \\
(67.6- \\
69.4)\end{array}$ & 483 & $\begin{array}{c}16.8 \\
(14.3- \\
19.4)\end{array}$ & 5,293 & $\begin{array}{c}27.0 \\
(25.6- \\
28.5)\end{array}$ & 14,654 & $\begin{array}{c}66.1 \\
(64.6- \\
67.6)\end{array}$ \\
\hline Rural & 23,073 & $\begin{array}{c}63.9 \\
(62.6- \\
65.1)\end{array}$ & 14,351 & $\begin{array}{c}74.3 \\
(73.0- \\
75.6)\end{array}$ & 14,302 & $\begin{array}{c}74.5 \\
(73.0- \\
75.9)\end{array}$ & 13,139 & $\begin{array}{c}36.4 \\
(35.2- \\
37.5)\end{array}$ & 17,379 & $\begin{array}{c}69.0 \\
(67.5- \\
70.5)\end{array}$ & 24,229 & $\begin{array}{c}32.4 \\
(31.6- \\
33.3)\end{array}$ & 8,361 & $\begin{array}{c}67.6 \\
(65.7- \\
69.6)\end{array}$ & 44 & $\begin{array}{l}13.1 \\
(6.6- \\
19.5)\end{array}$ & 773 & $\begin{array}{c}23.2 \\
(20.3- \\
26.1)\end{array}$ & 2,736 & $\begin{array}{c}65.3 \\
(61.9- \\
68.7)\end{array}$ \\
\hline $\begin{array}{l}\text { Urban to rural } \\
\text { prevalence } \\
\text { ratio* }\end{array}$ & $1.08^{\dagger}$ & $\begin{array}{c}(1.06- \\
1.10)\end{array}$ & $1.07^{\dagger}$ & $\begin{array}{c}(1.05- \\
1.09)\end{array}$ & $1.06^{\dagger}$ & $\begin{array}{c}(1.04- \\
1.08)\end{array}$ & $1.27^{\dagger}$ & $\begin{array}{c}(1.23- \\
1.31)\end{array}$ & $1.04^{\dagger}$ & $\begin{array}{c}(1.02- \\
1.06)\end{array}$ & $1.03^{\S}$ & $\begin{array}{c}(1.00- \\
1.06)\end{array}$ & 1.01 & $\begin{array}{c}(0.98- \\
1.05)\end{array}$ & 1.29 & $\begin{array}{c}(0.77- \\
2.16)\end{array}$ & $1.17^{\S}$ & $\begin{array}{c}(1.02- \\
1.34)\end{array}$ & 1.01 & $\begin{array}{c}(0.96- \\
1.07)\end{array}$ \\
\hline
\end{tabular}

Abbreviations: $\mathrm{Cl}=$ confidence interval; $\mathrm{FPL}=$ federal poverty level ; $\mathrm{HPV}=$ human papillomavirus.

* Generalized linear modeling was used to identify the prevalence ratio.

$+\mathrm{p}<0.01$.

$\S p<0.05$.

prevalence of the other four clinical preventive services were $<50 \%$, ranging from $16.5 \%$ for HPV vaccination to $45.8 \%$ for HIV testing. Being uninsured was associated with lower use of each of the 10 services, with PRs ranging from 1.03 for HIV testing to 2.99 for shingles vaccination. Persons with lower income had a lower prevalence for nine of 10 clinical preventive services compared with those with higher household incomes (eight of nine with $\mathrm{p}<0.01$ ). In contrast, HIV testing utilization was significantly higher among those with lower income. Among those eight services, the PRs for persons with higher versus lower income ranged from 1.08 to 1.88 . Persons living in rural areas used each of the recommended clinical preventive services less than those living in urban areas, with PRs for seven of these reaching statistical significance.

Use of clinical preventive services varied by state (Table 2). The variation in use differed substantially by type of service, with breast and cervical cancer screenings having the least cross-state variation among the six services asked by all states. Variation across states was widest for prevalence of HIV testing and pneumococcal vaccination use (3.1-fold and 2.5-fold, respectively).

The highest adjusted use was observed in the insured-higher income-urban group for six of the 10 services (all but HIV testing, diabetes screening, HPV vaccination, and tetanus vaccination). Insurance status was the factor most strongly associated with use of clinical preventive services, followed by income level and rurality, respectively. Uninsured persons used seven of the 10 clinical preventive services less frequently than those with insurance, regardless of income level and rurality. Among those with insurance, use of six of the 10 services was higher among persons with higher incomes, regardless of whether they lived in rural or urban counties (Figure) (Supplementary Figure, https://stacks.cdc.gov/view/cdc/104149) (Supplementary Table 1, https://stacks.cdc.gov/view/cdc/104150). 
TABLE 2. Percentage of adults who received recommended clinical preventive services, by jurisdiction — Behavioral Risk Factor Surveillance System, United States, 2018*

\begin{tabular}{|c|c|c|c|c|c|c|c|c|c|c|}
\hline \multirow[b]{2}{*}{ Jurisdiction } & \multicolumn{10}{|c|}{$\%(95 \% \mathrm{Cl})$} \\
\hline & $\begin{array}{l}\text { Colon cancer } \\
\text { screening, age } \\
50-75 \text { yrs }\end{array}$ & $\begin{array}{l}\text { Cervical cancer } \\
\text { screening } \\
\text { (women), age } \\
21-65 \text { yrs }\end{array}$ & $\begin{array}{l}\text { Breast cancer } \\
\text { screening } \\
\text { (within } 2 \text { yrs) } \\
\text { (women), age } \\
50-74 \text { yrs }\end{array}$ & $\begin{array}{l}\text { HIV testing } \\
\text { (ever), age } \\
18-65 \text { yrs }\end{array}$ & $\begin{array}{l}\text { Pneumococcal } \\
\text { vaccination } \\
\text { (ever), age } \\
\geq 65 \text { yrs }\end{array}$ & $\begin{array}{c}\text { Influenza } \\
\text { vaccination } \\
\text { (within } 1 \mathrm{yr} \text { ), } \\
\text { age } \geq 18 \mathrm{yrs}\end{array}$ & $\begin{array}{c}\text { Diabetes } \\
\text { screening } \\
\text { (within } 3 \text { yrs), } \\
\text { age } 40-70 \text { yrs }\end{array}$ & $\begin{array}{l}\text { HPV vaccination } \\
\text { (ever), age } \\
18-26 \text { yrs }\end{array}$ & $\begin{array}{c}\text { Zoster } \\
\text { (shingles) } \\
\text { vaccination } \\
\text { (ever), age } \\
\geq 50 \text { yrs }\end{array}$ & $\begin{array}{c}\text { Tetanus } \\
\text { vaccination } \\
\text { (within } 10 \mathrm{yrs} \text { ), } \\
\text { age } \geq 19 \text { yrs }\end{array}$ \\
\hline Alabama & $69.8(67.7-71.9)$ & $79.1(76.8-81.5)$ & $80.2(77.8-82.6)$ & $45.8(43.7-47.8)$ & $71.7(69.3-74.0)$ & $67.2(64.6-69.9)$ & $14.6(10.0-19.1)$ & NR & NR & NR \\
\hline Alaska & $59.6(55.8-63.4)$ & $76.3(72.4-80.3)$ & $67.3(62.0-72.5)$ & $45.4(42.2-48.5)$ & $64.2(59.6-68.8)$ & $33.8(31.2-36.3)$ & $63.5(58.9-68.2)$ & NR & NR & NR \\
\hline Arizona & $65.8(63.3-68.3)$ & $76.1(73.3-78.9)$ & $73.1(70.0-76.3)$ & $45.5(43.2-47.8)$ & $73.7(71.2-76.3)$ & $30.6(28.9-32.2)$ & $67.9(64.3-71.5)$ & NR & NR & NR \\
\hline Arkansas & $66.0(63.5-68.5)$ & $75.5(72.8-78.3)$ & $72.5(69.6-75.4)$ & $44.4(41.8-47.0)$ & $74.6(72.3-76.8)$ & $31.1(29.2-33.0)$ & NR & NR & NR & NR \\
\hline California & $70.1(68.2-72.0)$ & $78.9(77.1-80.7)$ & $81.2(78.8-83.5)$ & $49.0(47.6-50.5)$ & $68.7(65.8-71.6)$ & $32.4(31.2-33.6)$ & NR & NR & NR & NR \\
\hline Colorado & $67.8(66.0-69.5)$ & $76.6(74.7-78.6)$ & $71.1(68.8-73.4)$ & $41.5(40.0-43.1)$ & $77.1(75.2-79.0)$ & $36.6(35.3-37.8)$ & NR & NR & NR & NR \\
\hline Connecticut & $74.0(72.4-75.5)$ & $84.5(82.7-86.2)$ & $82.7(80.8-84.6)$ & $45.8(44.1-47.5)$ & $71.2(69.3-73.2)$ & $35.0(33.7-36.2)$ & NR & $25.6(20.5-30.7)$ & NR & NR \\
\hline Delaware & $72.0(69.8-74.2)$ & $82.4(79.9-84.9)$ & $83.8(81.4-86.3)$ & $48.6(46.2-50.9)$ & $72.6(69.9-75.3)$ & $38.2(36.3-40.0)$ & NR & NR & NR & NR \\
\hline $\mathrm{DC}$ & $72.3(69.9-74.8)$ & $83.7(80.8-86.5)$ & $79.6(76.7-82.4)$ & $76.7(74.4-78.9)$ & $70.8(68.0-73.7)$ & $44.2(42.1-46.3)$ & $72.0(68.5-75.4)$ & NR & NR & NR \\
\hline Florida & $69.6(67.4-71.8)$ & $79.4(77.1-81.6)$ & $81.2(79.0-83.4)$ & $52.8(50.8-54.9)$ & $67.2(64.7-69.8)$ & $30.7(29.2-32.1)$ & $70.6(67.3-73.8)$ & NR & NR & NR \\
\hline Georgia & $67.4(65.5-69.2)$ & $80.5(78.8-82.2)$ & $79.8(77.8-81.8)$ & $52.0(50.4-53.6)$ & $71.0(68.8-73.2)$ & $29.8(28.6-30.9)$ & $69.4(67.1-71.7)$ & NR & NR & NR \\
\hline Guam & $39.7(34.6-44.8)$ & $68.0(62.5-73.4)$ & $74.5(68.0-80.9)$ & $35.2(31.4-39.0)$ & $41.5(33.6-49.5)$ & $24.3(21.1-27.5)$ & $65.4(59.1-71.8)$ & $\mathrm{NR}$ & NR & NR \\
\hline Hawaii & $74.3(72.3-76.2)$ & $83.1(81.1-85.0)$ & $86.9(85.0-88.8)$ & $35.9(34.1-37.7)$ & $65.4(62.4-68.4)$ & $33.7(32.2-35.2)$ & $66.5(63.8-69.3)$ & $16.7(12.4-21.0)$ & NR & NR \\
\hline Idaho & $66.6(63.6-69.6)$ & $68.1(64.4-71.8)$ & $68.0(63.9-72.1)$ & $35.4(32.7-38.1)$ & $70.4(67.3-73.6)$ & $32.1(30.1-34.1)$ & $62.2(57.9-66.4)$ & $\mathrm{NR}$ & NR & NR \\
\hline Illinois & $65.8(63.4-68.2)$ & $78.5(76.2-80.8)$ & $78.4(75.4-81.5)$ & $38.7(36.8-40.7)$ & $68.7(65.7-71.7)$ & $32.2(30.6-33.7)$ & $\mathrm{NR}$ & NR & NR & NR \\
\hline Indiana & $67.1(65.2-69.0)$ & $78.7(76.6-80.8)$ & $76.4(74.2-78.6)$ & $41.2(39.3-43.1)$ & $71.8(69.8-73.9)$ & $28.5(27.1-29.8)$ & $69.6(67.1-72.1)$ & NR & NR & NR \\
\hline lowa & $70.9(69.3-72.4)$ & $79.5(77.7-81.3)$ & $80.7(78.8-82.5)$ & $30.6(29.2-31.9)$ & $76.1(74.3-77.9)$ & $40.6(39.4-41.8)$ & NR & NR & NR & NR \\
\hline Kansas & $66.5(65.0-68.1)$ & $74.4(72.5-76.3)$ & $74.2(72.3-76.1)$ & $34.5(33.0-35.9)$ & $75.9(74.3-77.5)$ & $36.0(34.9-37.2)$ & $\mathrm{NR}$ & NR & NR & NR \\
\hline Kentucky & $68.9(66.4-71.4)$ & $77.0(74.6-79.4)$ & $77.5(74.7-80.4)$ & $39.3(37.2-41.5)$ & $72.5(69.6-75.4)$ & $36.0(34.3-37.6)$ & $69.7(66.7-72.7)$ & NR & NR & NR \\
\hline Louisiana & $68.5(65.9-71.1)$ & $82.0(79.6-84.4)$ & $82.9(80.4-85.5)$ & $48.6(46.2-50.9)$ & $67.9(64.4-71.3)$ & $26.4(24.7-28.1)$ & $\mathrm{NR}$ & NR & NR & NR \\
\hline Maine & $74.9(73.2-76.5)$ & $82.4(80.5-84.4)$ & $80.8(78.9-82.8)$ & $39.5(37.5-41.5)$ & $76.7(75.1-78.4)$ & $32.4(31.0-33.8)$ & $72.3(70.1-74.5)$ & NR & NR & NR \\
\hline Maryland & $71.5(70.1-72.9)$ & $81.8(80.2-83.5)$ & $81.1(79.5-82.7)$ & $55.4(54.0-56.9)$ & $75.3(73.6-76.9)$ & $39.5(38.3-40.6)$ & $72.9(71.1-74.6)$ & NR & NR & NR \\
\hline Massachusetts & 75.9 (73.9-77.9) & $82.9(80.8-85.0)$ & $86.2(84.0-88.3)$ & $44.6(42.6-46.5)$ & $72.4(69.7-75.1)$ & $37.1(35.6-38.6)$ & NR & NR & NR & NR \\
\hline Michigan & $73.8(72.2-75.4)$ & $82.7(81.0-84.3)$ & $80.1(78.1-82.1)$ & $45.8(44.3-47.3)$ & $73.8(71.7-75.9)$ & $32.3(31.2-33.5)$ & NR & NR & NR & NR \\
\hline Minnesota & $72.5(71.2-73.7)$ & $81.0(79.7-82.3)$ & $82.2(80.7-83.6)$ & $35.1(34.0-36.2)$ & $72.5(71.0-74.1)$ & $39.7(38.8-40.6)$ & NR & NR & NR & NR \\
\hline Mississippi & $62.0(59.7-64.4)$ & $80.4(78.2-82.5)$ & $71.0(68.2-73.9)$ & $47.3(45.1-49.4)$ & $68.6(65.9-71.3)$ & $32.7(31.1-34.3)$ & $64.1(61.2-66.9)$ & $15.7(10.9-20.6)$ & $\mathrm{NR}$ & $57.2(55.4-59.1)$ \\
\hline Missouri & $69.2(66.9-71.5)$ & $77.5(74.8-80.3)$ & $75.3(72.4-78.1)$ & $39.4(37.2-41.7)$ & $73.9(71.6-76.3)$ & $36.5(34.8-38.2)$ & $70.3(67.3-73.4)$ & $17.1(11.7-22.5)$ & $27.0(25.2-28.8)$ & $71.2(69.3-73.0)$ \\
\hline Montana & $63.3(60.8-65.8)$ & $73.5(70.4-76.5)$ & $73.7(70.5-77.0)$ & $37.4(35.2-39.6)$ & $73.4(70.6-76.2)$ & $35.7(33.9-37.4)$ & $\mathrm{NR}$ & NR & $\mathrm{NR}$ & NR \\
\hline Nebraska & $68.1(66.5-69.7)$ & $78.0(76.2-79.7)$ & $75.2(73.2-77.3)$ & $29.6(28.2-31.0)$ & $76.4(74.8-78.1)$ & $39.4(38.1-40.6)$ & NR & NR & NR & NR \\
\hline Nevada & $59.9(56.0-63.9)$ & $77.4(73.6-81.2)$ & $72.3(67.9-76.8)$ & $47.2(44.1-50.3)$ & $68.1(63.5-72.7)$ & $32.6(30.1-35.0)$ & NR & NR & NR & NR \\
\hline New Hampshire & $74.1(72.1-76.1)$ & 82.9 & $82.8(80.6-85.1)$ & $42.0(39.7-44.4)$ & $78.5(76.4-80.6)$ & $33.3(31.6-35.0)$ & NR & NR & NR & NR \\
\hline New Jersey & $66.6(62.3-70.9)$ & $78.8(74.4-83.2)$ & $80.8(76.1-85.5)$ & $49.8(46.3-53.3)$ & $67.8(61.5-74.1)$ & $38.1(35.3-41.0)$ & $78.4(73.8-83.0)$ & $18.8(10.5-27.1)$ & NR & NR \\
\hline New Mexico & $63.8(61.5-66.1)$ & 77.1 (74.7-79.4) & $71.6(68.8-74.5)$ & $38.4(36.4-40.4)$ & $70.9(68.1-73.8)$ & $34.3(32.7-35.9)$ & $71.4(68.6-74.3)$ & $\mathrm{NR}$ & NR & NR \\
\hline New York & $68.9(67.5-70.4)$ & $81.5(80.1-82.9)$ & $82.1(80.4-83.9)$ & $57.0(55.8-58.1)$ & $63.8(61.6-66.0)$ & $28.0(27.1-28.9)$ & $65.2(63.4-67.0)$ & NR & NR & NR \\
\hline North Carolina & $71.0(68.5-73.5)$ & $80.3(78.0-82.7)$ & $79.3(76.1-82.5)$ & $52.1(49.9-54.2)$ & $76.2(73.0-79.5)$ & $41.7(39.9-43.5)$ & $72.0(68.8-75.2)$ & NR & NR & NR \\
\hline North Dakota & $66.5(64.3-68.7)$ & $75.0(71.9-78.1)$ & $78.9(76.2-81.6)$ & $33.6(31.4-35.8)$ & $75.0(72.8-77.2)$ & $40.0(38.2-41.9)$ & $65.3(62.4-68.2)$ & NR & NR & NR \\
\hline Ohio & $66.6(64.9-68.3)$ & $79.0(77.1-80.8)$ & $77.4(75.4-79.3)$ & $39.6(38.0-41.3)$ & $74.1(72.3-75.9)$ & $35.2(33.9-36.4)$ & NR & NR & NR & NR \\
\hline Oklahoma & $62.1(59.7-64.5)$ & $73.5(71.0-76.1)$ & $74.2(71.4-77.0)$ & $36.4(34.2-38.5)$ & $74.8(72.4-77.2)$ & $38.1(36.5-39.8)$ & NR & NR & NR & NR \\
\hline Oregon & $71.7(69.5-73.9)$ & $78.0(75.7-80.3)$ & $77.9(75.3-80.6)$ & $45.7(43.8-47.6)$ & $77.1(74.4-79.9)$ & $30.6(29.1-32.1)$ & $67.7(64.8-70.5)$ & NR & NR & NR \\
\hline Pennsylvania & $71.3(69.1-73.5)$ & $77.3(74.8-79.8)$ & $78.6(75.8-81.5)$ & $41.4(39.4-43.3)$ & 74.7 (71.9-77.5) & $40.3(38.7-41.9)$ & NR & NR & NR & NR \\
\hline Puerto Rico & $55.7(53.2-58.3)$ & $81.6(79.5-83.7)$ & $83.5(81.0-86.0)$ & $61.5(59.4-63.6)$ & $31.1(28.0-34.2)$ & $25.8(24.2-27.3)$ & $85.5(83.3-87.7)$ & NR & NR & NR \\
\hline Rhode Island & 75.1 (72.9-77.3) & $83.9(81.5-86.4)$ & $87.0(85.0-89.0)$ & $48.4(46.0-50.8)$ & $74.6(72.1-77.1)$ & $37.1(35.2-38.9)$ & NR & NR & NR & NR \\
\hline South Carolina & $70.3(68.7-71.9)$ & $78.7(76.8-80.6)$ & $77.2(75.2-79.1)$ & $44.3(42.6-46.0)$ & $73.4(71.7-75.1)$ & $35.5(34.3-36.8)$ & $69.7(67.4-71.9)$ & NR & NR & NR \\
\hline South Dakota & $68.4(65.6-71.2)$ & $72.9(68.9-76.9)$ & $81.7(78.6-84.8)$ & $32.0(29.4-34.6)$ & 76.5 (73.7-79.4) & $35.3(33.2-37.4)$ & $63.7(59.9-67.4)$ & NR & NR & NR \\
\hline Tennessee & $68.3(65.7-70.8)$ & $78.9(76.2-81.7)$ & $76.3(73.1-79.5)$ & $42.5(40.1-44.9)$ & $74.2(71.3-77.0)$ & $28.6(26.9-30.3)$ & $69.0(65.7-72.2)$ & $19.8(12.3-27.2)$ & $24.6(22.5-26.8)$ & NR \\
\hline Texas & $59.3(55.9-62.8)$ & $78.2(75.5-81.0)$ & $74.9(70.4-79.5)$ & $47.1(44.8-49.5)$ & $71.1(67.2-74.9)$ & $26.4(24.6-28.1)$ & $61.6(57.2-65.9)$ & $13.7(9.3-18.1)$ & $25.7(23.2-28.2)$ & $62.7(60.3-65.1)$ \\
\hline Utah & $69.5(67.7-71.3)$ & $73.0(71.2-74.9)$ & $72.3(69.8-74.8)$ & $24.7(23.5-25.9)$ & 73.7 (71.6-75.9) & $32.3(31.2-33.5)$ & $\mathrm{NR}$ & NR & $\mathrm{NR}$ & NR \\
\hline Vermont & $71.2(69.2-73.1)$ & $78.4(75.8-81.0)$ & 76.4 (73.9-78.9) & $44.1(41.8-46.3)$ & $74.5(72.2-76.9)$ & $37.2(35.4-38.9)$ & NR & NR & NR & NR \\
\hline Virginia & $69.3(67.5-71.1)$ & $83.6(81.9-85.3)$ & $81.1(79.1-83.1)$ & $47.3(45.6-49.0)$ & $73.6(71.5-75.7)$ & $38.9(37.6-40.3)$ & $71.3(69.1-73.6)$ & NR & $30.0(28.5-31.5)$ & 73.5 (72.1-74.8) \\
\hline Washington & $70.8(69.3-72.4)$ & $76.3(74.6-78.1)$ & $74.8(72.8-76.8)$ & $44.9(43.4-46.3)$ & $77.7(76.2-79.3)$ & $38.4(37.3-39.5)$ & $67.0(64.9-69.1)$ & NR & NR & NR \\
\hline West Virginia & $67.4(65.3-69.5)$ & $78.0(75.6-80.4)$ & $75.1(72.3-77.9)$ & $35.1(33.0-37.2)$ & $73.0(70.6-75.4)$ & $42.6(40.9-44.3)$ & $73.4(70.7-76.0)$ & NR & NR & NR \\
\hline Wisconsin & $74.0(71.6-76.4)$ & 79.5 (76.9-82.0) & $77.8(74.8-80.9)$ & $33.9(31.6-36.1)$ & 74.7 (71.7-77.7) & $29.9(28.1-31.6)$ & $68.6(65.6-71.7)$ & NR & NR & NR \\
\hline Wyoming & $57.7(55.3-60.1)$ & $73.2(70.4-76.1)$ & $68.0(64.9-71.0)$ & $38.0(35.8-40.3)$ & $69.8(67.2-72.3)$ & $31.0(29.4-32.7)$ & $60.2(57.0-63.3)$ & NR & NR & NR \\
\hline
\end{tabular}

Abbreviations: $\mathrm{Cl}=$ confidence interval; $\mathrm{DC}=$ District of Columbia; $\mathrm{HPV}=$ human papillomavirus; $\mathrm{NR}=$ not reported.

* 50 states, DC, Puerto Rico, and Guam were included in the analysis of cancer screenings, HIV testing, pneumococcal vaccination, and influenza vaccination; 28 states, DC, Puerto Rico, and Guam were included in the analysis of diabetes screening; eight states were included in the analysis of HPV vaccination; and four states were included in the analysis of both zoster (shingles) and tetanus vaccinations. 
FIGURE. Adjusted prevalence ratios of use of selected clinical preventive services, ${ }^{*}$ by health insurance status, family income level, and rurality — Behavioral Risk Factor Surveillance System, United States, 2018

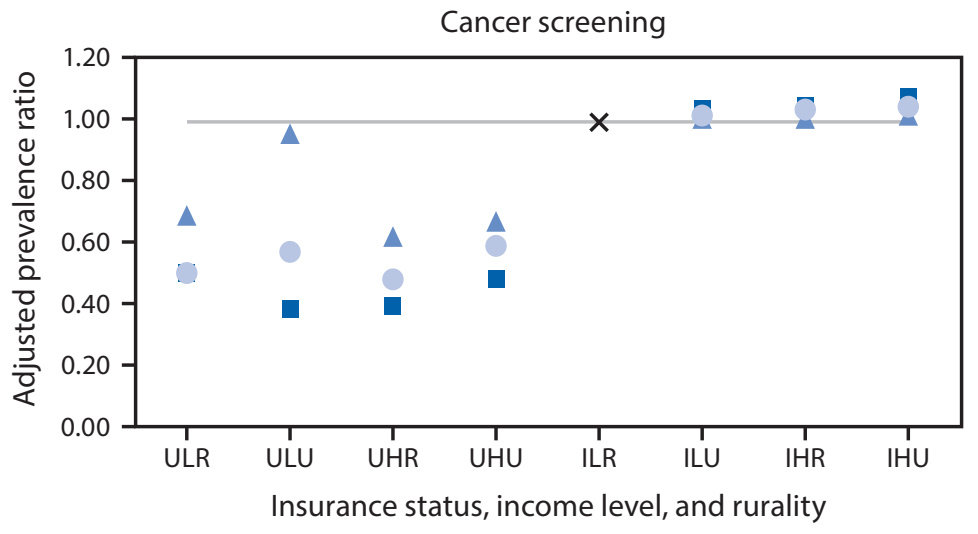

Influenza vaccination

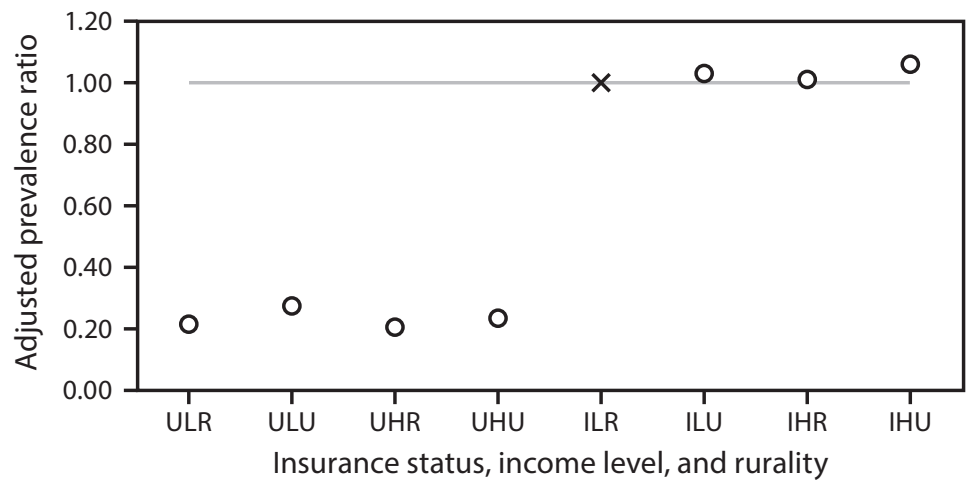

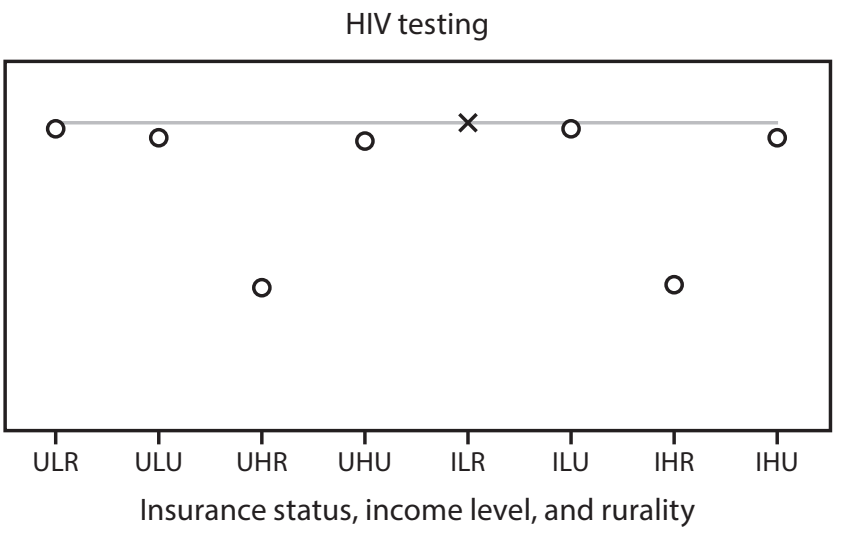

Diabetes screening

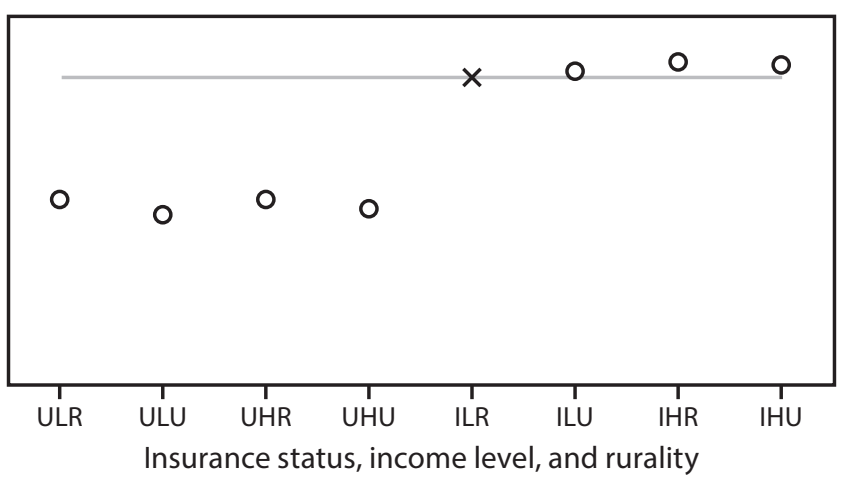

- Colon, $\mathrm{p}<0.05 \Delta$ Cervical, $\mathrm{p}<0.05 \quad$ Breast, $\mathrm{p}<0.05 \quad \times$ Reference group $\mathrm{O} \mathrm{p}<0.05 \quad$ Adjusted prevalence ratio $=1$

Abbreviations: $\mathrm{HPV}=$ human papillomavirus; $\mathrm{IHR}=$ insured and higher income and rural; $\mathrm{IHU}=$ insured and higher income and urban; $\mathrm{ILR}=$ insured and lower income and rural; ILU = insured and lower income and urban; UHR = uninsured and higher income and rural; UHU = uninsured and higher income and urban; ULR = uninsured and lower income and rural; ULU = uninsured and lower income and urban.

* Adjusted by age, sex (except for cervical cancer screening and breast cancer screening), race/ethnicity, education level, marital status, self-reported health status, and state. Similar findings were observed in pneumococcal, HPV, zoster (shingles), and tetanus vaccinations (panels available in Supplementary Figure, https:// stacks.cdc.gov/view/cdc/104149).

\section{Discussion}

In 2018, use of nine recommended clinical preventive services was lower among persons without insurance, those with lower income, and those living in rural communities, whereas use of HIV testing was higher among persons with lower income. Geographic variation in use of clinical preventive services existed across states. These differences varied by type of services, with variation being greatest for HIV testing use. Insurance status had the strongest association with use of clinical preventive services followed by income and rurality.

Use of nearly all recommended clinical preventive services was higher in 2018 than it was during 2011-2012 (3,4). These results were consistent with previous studies, which showed that the prevalence of use of clinical preventive services was lower among persons who were uninsured, lived in households with lower income, and lived in nonmetropolitan areas (3-5).
Geographic variation was also consistent with previous studies, which suggests that state-level variation could be used to identify state- and locality-specific strategies to increase use of clinical preventive services $(6,8)$. In addition, policies that address health insurance coverage and benefits or reduce specific barriers to care for persons with lower income or living in rural areas could potentially be effective at increasing use of clinical preventive services. The finding that use of HIV testing was higher among persons of lower income was consistent with previous studies $(2,3)$ and might reflect the success of a testing strategy that focused HIV screening efforts in communities that are disproportionately comprised of persons of lower income (9). Fear and misperceptions about HIV risk and the testing process itself might be additional barriers to increasing HIV testing (10). 


\section{Summary}

What is already known on this topic?

Ongoing federal and state health reform efforts, particularly the Patient Protection and Affordable Care Act, have affected use of clinical preventive services in the United States.

What is added by this report?

Analysis of 2018 Behavioral Risk Factor Surveillance System data indicated use increased for selected recommended clinical preventive services; however, use of nine of the 10 services examined was lower among the uninsured, those with lower income, and those living in rural communities. Among those factors examined, insurance status had the strongest association with use of clinical preventive services, followed by income level and rurality.

What are the implications for public health practice?

Understanding factors influencing use of clinical preventive services can potentially help decision makers better identify policies to increase their use including strategies to increase insurance coverage.

The findings in this report are subject to at least six limitations. First, the analysis was based on self-reported use data, which could be subject to recall and social desirability bias. Second, use of some services as measured by BRFSS was not entirely aligned with the recommendations; BRFSS questions, recommendations, and important distinctions are provided (Supplementary Table 2, https://stacks.cdc.gov/ view/cdc/104148). Third, FPL was estimated based on the categorical income value provided by BRFSS rather than a precise estimate of household income. Fourth, whether BRFSS participants received services from in-network providers could not be determined, nor could whether survey participants were enrolled in insurance plans subject to ACA requirements to provide clinical preventive services without cost-sharing be determined (1). Therefore, use among the insured group was potentially underestimated compared with a sample comprised entirely of persons with ACA-compliant plans. Fifth, this is a cross-sectional study, and causal relationship cannot be determined even when relevant confounders are adequately controlled. Finally, only a limited number of states participated in BRFSS optional modules for diabetes screening and for HPV, shingles and tetanus vaccinations, and so data might not be nationally representative of prevalence, even though the results were consistent with previous studies $(3,4)$.

As the health care policy landscape continues to shift, understanding factors associated with use of recommended clinical preventive services could help decision makers better identify policy levers to increase use of clinical preventive services. The ongoing monitoring of trends could improve understanding of how modifiable factors affect use of clinical preventive services, especially during the pandemic, because a decrease in use of routine vaccinations was observed. Although insurance status, income level, rurality, and state of residence appear to be associated with use, examining other barriers could also help better identify strategies to achieve public health goals.

Corresponding author: Suhang Song, suhangsong@gmail.com, 571-267-9586.

\footnotetext{
${ }^{1}$ Policy Research, Analysis, and Development Office, Office of the Associate Director for Policy and Strategy, CDC; ${ }^{2}$ The Oak Ridge Institute for Science and Education, Oak Ridge, Tennessee.
}

All authors have completed and submitted the International Committee of Medical Journal Editors form for disclosure of potential conflicts of interest. No potential conflicts of interest were disclosed.

\section{References}

1. Fox JB, Shaw FE. Clinical preventive services coverage and the Affordable Care Act. Am J Public Health 2015;105:e7-10. PMID:25393173 https://doi.org/10.2105/AJPH.2014.302289

2. Okoro CA, Zhao G, Fox JB, Eke PI, Greenlund KJ, Town M. Surveillance for health care access and health services use, adults aged 18-64 years - Behavioral Risk Factor Surveillance System, United States, 2014. MMWR Surveill Summ 2017;66(No. SS-7). PMID:28231239 https://doi.org/10.15585/mmwr.ss6607a1

3. Fox JB, Shaw FE. Relationship of income and health care coverage to receipt of recommended clinical preventive services by adults-United States, 2011-2012. MMWR Morb Mortal Wkly Rep 2014;63:666-70. PMID:25102414 https:/www.cdc.gov/mmwr/preview/mmwrhtml/ mm6331a2.htm?s_cid=mm6331a2_w

4. Fox JB, Shaw FE. Receipt of selected clinical preventive services by adults-United States, 2011-2012. MMWR Morb Mortal Wkly Rep 2015;64:738-42. PMID:26182191 https://www.cdc.gov/mmwr/ preview/mmwrhtml/mm6427a2.htm

5. Joseph DA, King JB, Dowling NF, Thomas CC, Richardson LC. Vital signs: colorectal cancer screening test use-United States, 2018. MMWR Morb Mortal Wkly Rep 2020;69:253-9. PMID:32163384 https://doi. org/10.15585/mmwr.mm6910a1

6. Henley SJ, King JB, German RR, Richardson LC, Plescia M. Surveillance of screening-detected cancers (colon and rectum, breast, and cervix) United States, 2004-2006. MMWR Surveill Summ 2010;59(No. SS-9). PMID:21102407

7. CDC. Statistical brief on the health care access module, 2013 and 2014. Atlanta, GA: US Department of Health and Human Services, CDC; 2015. https://www.cdc.gov/brfss/data_documentation/pdf/20132014_hcs.pdf

8. Nelson DE, Bland S, Powell-Griner E, et al. State trends in health risk factors and receipt of clinical preventive services among US adults during the 1990s. JAMA 2002;287:2659-67. PMID:12020301 https://doi. org/10.1001/jama.287.20.2659

9. Pellowski JA, Kalichman SC, Matthews KA, Adler N. A pandemic of the poor: social disadvantage and the U.S. HIV epidemic. Am Psychol 2013;68:197-209. PMID:23688088 https://doi.org/10.1037/a0032694

10. CDC. CDC fact sheet: HIV testing in the United States. Atlanta, GA: US Department of Health and Human Services, CDC; 2016. https:// www.cdc.gov/nchhstp/newsroom/docs/factsheets/hiv-testing-us-508.pdf 